\title{
Treatment Failure of Continuous Positive Airway Pressure with a Full Face Mask, Reversed with a Nasal Mask
}

\author{
Jee Hyun Kim ${ }^{1}$, Jae Wook Cho ${ }^{2}$ \\ ${ }^{1}$ Department of Neurology, Dankook University College of Medicine, Dankook University Hospital, Cheonan, \\ ${ }^{2}$ Department of Neurology, Research Institute for Convergence of Biomedical Science and Technology, Pusan National University \\ Yangsan Hospital, Yangsan, Korea
}

Received June 6, 2016

Revised June 26, 2016

Accepted September 26, 2016

Address for correspondence Jee Hyun Kim, MD, PhD

Department of Neurology,

Dankook University

College of Medicine,

Dankook University Hospital,

201 Manghyang-ro, Dongnam-gu,

Cheonan 31116, Korea

Tel: +82-41-550-3292

Fax: +82-41-550-6425

E-mail: fever26@gmail.com
Although a nasal mask is a standard interface for continuous positive airway pressure (CPAP) treatment for obstructive sleep apnea (OSA), severe mouth breathing during sleep often leads to the use of a full face mask which covers the nose and mouth. Herein, we present a case of a patient with uncontrolled severe OSA with CPAP and a full face mask, who subsequently shows dramatic improvement of OSA with a nasal mask and lower CPAP pressure.

J Sleep Med 2016;13(2):67-69

Key Words: Nasal mask, Full face mask, Continuous positive airway pressure.

Mouth breathing is a common phenomenon in patients with obstructive sleep apnea (OSA), ${ }^{1,2}$ which can be decreased with the treatment of OSA and/or the intervention of nasal obstructions. ${ }^{2}$ During continuous positive airway pressure (CPAP) treatment, mouth breathing results in mouth dryness and disruptive noises from mask leak. Full face masks are frequently recommended alternatives for patients who have excessive mask leaks from mouth breathing. We present a case in which CPAP failed to alleviate OSA with a full face mask but succeeded with a nasal mask.

\section{Case Report}

A 65-year-old man was referred to the sleep clinic for the evaluation of heavy snoring and frequent witnessed sleep apnea. The patient had history of right middle cerebral infarction and hypertension. On neurological examination, he had a left hemiplegia and severe dysarthria. The body mass index was $23.4 \mathrm{~kg} / \mathrm{m}^{2}$. An overnight polysomnogram demonstrated severe OSA with apnea-hypopnea index (AHI), 70.7/h. The

This is an Open Access article distributed under the terms of the Creative Commons Attribution Non-Commercial License (http://creativecommons.org/licenses/by-nc/3.0) which permits unrestricted non-commercial use, distribution, and reproduction in any medium, provided the original work is properly cited. lowest oxygen desaturation was $83 \%$. Full night CPAP titration was performed with a nasal mask and a chin strap, and it revealed the optimal pressure of $7 \mathrm{cmH}_{2} \mathrm{O}$. The patient was recommended using a nasal mask for CPAP treatment, but switch to a full face mask from the vendor due to significant mouth breathing. He tried CPAP with a full face mask for 4 months. His wife witnessed frequent episodes of apnea followed by brief awakenings with vigorous body movements and gasping despite daily CPAP use. The device usage data showed uncontrolled OSA with high AHI (mean 65.7/h) and high leak value (Table 1 ).

Empirically increasing CPAP pressure up to $15 \mathrm{cmH}_{2} \mathrm{O}$ did not eliminate OSA. He was recommended to use a nasal mask with a chin strap at $8 \mathrm{cmH}_{2} \mathrm{O}$ after the repeated CPAP titration (Table 2). At the follow up visit, the CPAP usage data demonstrated that his OSA had been perfectly controlled (AHI 1.7/h) (Table 1). His wife also reported clinical improvement of his fragmented and restless sleep.

\section{Discussion}

Traditionally, a full face mask is recommended when 1) the patient exhibits the presence of unacceptable mouth leaks, 
Table 1. Comparison of CPAP usage data with different masks

\begin{tabular}{lcc}
\hline \multicolumn{1}{c}{ Mask } & Full face mask & Nasal mask \\
\hline CPAP pressure $\left(\mathrm{cmH}_{2} \mathrm{O}\right)$ & $7 ; 8 ; 9$ & 8 \\
Apnea-hypopnea index $(/ \mathrm{h})$ & $67 ; 62.2 ; 64.6$ & 1.7 \\
90th percentile leak $(\mathrm{l} / \mathrm{min})$ & $76.4 ; 81 ; 85.6$ & 53.6 \\
Usage $(\mathrm{h})$ & $8.7 ; 8.2 ; 8.6$ & 8.2 \\
\hline
\end{tabular}

CPAP: continuous positive airway pressure

Table 2. The results of the follow-up CPAP polysomnography

\begin{tabular}{|c|c|c|c|c|}
\hline \multicolumn{5}{|c|}{ Sleep parameter } \\
\hline TST (min) & 373.5 & \multicolumn{2}{|c|}{ Sleep efficiency (\%) } & 74.8 \\
\hline SL (min) & 86 & \multicolumn{2}{|c|}{ REM SL (min) } & 35 \\
\hline N1/N2/N3/REM (\%) & $12.4 / 65.5 / 0 / 22.1$ & \multicolumn{2}{|c|}{ Supine sleep time (\%) } & 100 \\
\hline Arousal index $(/ \mathrm{h})$ & 6.4 & \multicolumn{2}{|c|}{ WASO (\%) } & 9.7 \\
\hline \multicolumn{5}{|c|}{ CPAP titration data } \\
\hline Pressure $\left(\mathrm{cmH}_{2} \mathrm{O}\right)$ & Time (min) & Body position & $\mathrm{AHI}(/ \mathrm{h})$ & Snoring \\
\hline 4 & 16.3 & Supine & 40.6 & Mild \\
\hline 5 & 12.1 & Supine & 54.6 & Mild \\
\hline 6 & 11.2 & Supine & 37.7 & Mild \\
\hline 7 & 297.1 & Supine & 1.2 & Mild \\
\hline 8 & 35.4 & Supine & 0 & None \\
\hline
\end{tabular}

TST: total sleep time, SL: sleep latency, REM: rapid eye movement, CPAP: continuous positive airway pressure, WASO: wakefulness after sleep onset, AHI: apnea-hypopnea index

preventing maintenance of adequate positive pressure or causing repeated arousals or throat discomfort due to dryness, 2) the patient is unable to breathe nasally due to nasal congestion. ${ }^{3}$

For the patient in the current case, a full face mask failed to deliver adequate CPAP treatment, and it shows that the mask type can affect the treatment outcome significantly.

In terms of the effectiveness of CPAP delivered by different mask types, previous studies presented different results. Although the consequent CPAP titration night studies showed no difference of pressure between a full face mask and a nasal mask within patients, ${ }^{4}$ a more recent study demonstrated that a full face mask group needed higher pressure than either a nasal mask or a nasal pillow, especially for patients with high AHI. ${ }^{5}$

Previous work investigating the upper airway-flow relationship in OSA demonstrated that a full face mask did not induce enough inspiratory flow to open airway. ${ }^{6}$ They assumed that CPAP transmitted via face mask increased $P_{\text {us }}$ (upstream pressure) and $\mathrm{P}_{\text {crit }}$ (critical pressure) simultaneously and resulted in no change in the flow gradient $\left(\mathrm{P}_{\text {us }}-\mathrm{P}_{\text {crit }}\right){ }^{6}$ In addition, the endoscopic examination during CPAP titration anecdotally showed posterior tongue displacement by the pressure delivered through the mouth via a full face mask. ${ }^{7}$ More recently, one case series described paradoxical worsening of upper airway obstruction by facial masks and showed the im- provement with nasal masks, supporting our case. ${ }^{8}$

In the current case, the 90th percentile leak level by CPAP was still high with a nasal mask although it was much lower than with a full face mask. Therefore, the treatment failure by a full face mask could be due not only to a high leak but also to the failure of creating enough pressure gradients to open the collapsed airway. Pharyngeal muscle weakness resulted from cerebral infarction could be another factor. Recently, there was a case report showing noninvasive ventilation with a full face mask which induced obstructive events in a patient with amyotrophic lateral sclerosis. This suggests that neuromuscular weakness could worsen or induce OSA in patients using CPAP with a full face mask. ${ }^{9}$

This case suggests that a full face mask is not always a good alternative choice for severe mouth breathers and might lead to inadequate treatment of OSA or paradoxical worsening of OSA. Re-trying a nasal mask with an effort to keep the mouth closed using a chinstrap or chin-up tapes would be helpful for mouth breathers, especially in case of treatment failure by a full face mask.

\section{REFERENCES}

1. Lee SM, Lee YJ, Kim JH. Common side effects and compliance with nasal continuous positive airway pressure in Korean OSA patients: short-term follow up. J Korean Sleep Res Soc 2010;7:1-7.

2. Bachour A, Maasilta P. Mouth breathing compromises adherence to na- 
sal continuous positive airway pressure therapy. Chest 2004;12:1248-1254.

3. Sanders MH, Kern NB, Stiller RA, Strollo PJ Jr, Martin TJ, Atwood CW Jr. CPAP therapy via oronasal mask for obstructive sleep apnea. Chest 1994;106:774-779.

4. Teo M, Amis T, Lee S, Falland K, Lambert S, Wheatley J. Equivalence of nasal and oronasal masks during initial CPAP titration for obstructive sleep apnea syndrome. Sleep 2011;34:951-955.

5. Ebben MR, Oyegbile T, Pollak CP. The efficacy of three different mask styles on a PAP titration night. Sleep Med 2012;13:645-649.

6. Smith PL, Wise RA, Gold AR, Schwartz AR, Permutt S. Upper airway pressure-flow relationships in obstructive sleep apnea. J Appl Physiol (1985) 1988;64:789-795.
7. Schorr F, Genta PR, Gregório MG, Danzi-Soares NJ, Lorenzi-Filho G. Continuous positive airway pressure delivered by oronasal mask may not be effective for obstructive sleep apnoea. Eur Respir J 2012;40:503-505.

8. Ng JR, Aiyappan V, Mercer J, et al. Choosing an oronasal mask to deliver continuous positive airway pressure may cause more upper airway obstruction or lead to higher continuous positive airway pressure requirements than a nasal mask in some patients: a case series. J Clin Sleep Med 2016;12:1227-1232.

9. Vrijsen B, Buyse B, Belge C, Testelmans D. Upper airway obstruction during noninvasive ventilation induced by the use of an oronasal mask. J Clin Sleep Med 2014;10:1033-1035. 